\title{
RDUS
}

Revue de DROIT

UNIVERSITÉ DE SHERBROOKE

Titre : $\quad$ LA BONNE FOI DES PARTIES AU CONTRAT À TITRE ONÉREUX DANS L'ACTION EN INOPPOSABILITÉ : RÉFORME OU STATU QUO?

Auteur(s) : $\quad$ François TÔTH, Nathalie VÉZINA

Revue: $\quad$ RDUS, 1992-1993, volume 23, numéro 1

Pages: $\quad 215-233$

ISSN : $\quad 0317-9656$

Éditeur : $\quad$ Université de Sherbrooke. Faculté de droit.

URI : $\quad$ http://hdl.handle.net/11143/13387

DOI : $\quad$ https://doi.org/10.17118/11143/13387 
Page vide laissée intentionnellement. 


\section{LA BONNE FOI DES PARTIES AU CONTRAT À TITRE ONÉREUX DANS L'ACTION EN INOPPOSABILITÉ : RÉFORME OU STATU QUO?}

par François TÔTH*

Nathalie VÉZINA**

\section{SOMMAIRE}

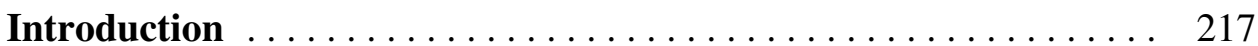

I. L'intention frauduleuse du débiteur . . . . . . . . . . 219

A. Le droit antérieur $\ldots \ldots \ldots \ldots \ldots \ldots \ldots \ldots \ldots$

B. Le droit nouveau ................... 223

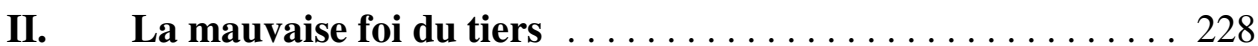

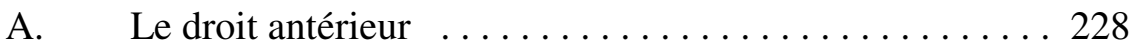

B. Le droit nouveau ................... 231

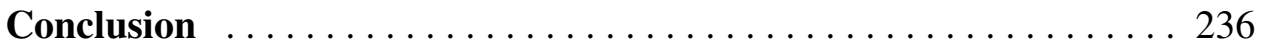



dans l'action en inopposabilité :

(1992) 23 R.D.U.S. réforme ou statu quo? 
(1992) 23 R.D.U.S.

La bonne foi des parties au contrat à titre onéreux dans l'action en inopposabilité :

\section{Introduction}

L'action paulienne, rebaptisée «action en inopposabilité» à l'occasion de la réforme du Code civil, peut s'avérer fort utile au créancier désireux de sauvegarder le patrimoine de son débiteur. Considéré sous un autre angle, ce recours se présente plutôt comme une immixtion grave dans les affaires du débiteur et des tiers qui acceptent de contracter avec lui. L'un des aspects les plus intéressants de l'action en inopposabilité réside donc dans la recherche d'un équilibre entre les intérêts des différentes parties au litige ${ }^{1}$. Or, en matière de contrats à titre onéreux, l'identification de ce point d'équilibre passe traditionnellement par la notion de bonne foi des parties à l'acte attaqué.

Il est vrai que d'autres éléments peuvent entrer en ligne de compte pour juger du droit d'un créancier d'attaquer l'acte intervenu entre son débiteur et un tiers. À première vue, toutefois, le droit nouveau ne semble pas entraîner de bouleversements majeurs à l'égard de ces autres facteurs. Tout au plus, le législateur a-t-il veillé à préciser ou nuancer les conditions relatives au préjudice subi par le créancier-demandeur ${ }^{2}$, au délai pour intenter l'action ${ }^{3}$ ou encore aux

1. Pour des considérations générales sur cette question d'équilibre entre les droits des parties en présence, voir: G. Trudel, Traité de droit civil du Québec, t. 7, Montréal, Wilson et Lafleur, 1946, p. 435-437.

2. Le Code fournit désormais des exemples de situations d'actes commis en fraude des droits du créancier et lui causant un préjudice (art. 1631 C.c.Q.).

Il faut préciser que les notions de préjudice et d'acte frauduleux sont indissociables. Elles représentent la cause et l'effet - la volonté du débiteur de diminuer son patrimoine, gage commun des créanciers, et le préjudice qui en découle pour ces derniers. Il ne fait pas de doute, dans le contexte de l'action en inopposabilité, que seule la fraude efficace, c'est-à-dire qui cause un préjudice à un ou plusieurs des créanciers, est susceptible d'être sanctionnée. Langelier explique que le geste du débiteur doit comporter à la fois l'animus et l'eventus, c'est-à-dire l'intention de frauder et le préjudice causé au créancier, pour que l'action paulienne soit accueillie: F. Langelier, Cours de droit civil de la Province de Québec, t. 3 , Montréal, Wilson et Lafleur, 1907, p. 436; voir aussi: C.-B.-M. Toullier, Le droit civil français suivant l'ordre du Code, t. 6, Paris, Jules Renouard, 1830, $\mathrm{n}^{\circ} 348$.

3. Le délai dont dispose le créancier pour prendre action est toujours limité à un an. Le législateur formule le point de départ de ce délai en des termes quelques peu différents, puisque le Code précise désormais que le délai court à partir de la connaissance du préjudice qui résulte de cet acte (art. 1635 C.c.Q.; comp. art. 1040 C.c.B.-C.), rejoignant ainsi l'interprétation jurisprudentielle sur la question; à cet effet, voir notamment: Crépeault c. Ville d'Anjou, C.A. Montréal, 500-09-001471-820, 15 février 1985, J.E. 85-233.

Ce délai s'impose principalement pour des motifs d'ordre public. D'ailleurs, il est généralement cité à titre d'exemple de délai préfix. Si une telle qualification appelait certaines réserves dans le droit antérieur (notamment quant à la possibilité d'invoquer une suspension comme pour un délai de prescription), le texte de l'art. 1635 C.c.Q. ne permet plus d'en douter; sur cette question, voir: J.-L. Baudouin, Les obligations, $4^{\mathrm{e}}$ éd., Cowansville, Éditions Yvon Blais, 1993, nº 671. 
qualités de la créance invoquée au soutien du recours ${ }^{4}$ Quant aux effets de l'action en inopposabilité (art. 1631 C.c.Q.), le Code civil du Québec se borne à codifier la position déjà énoncée en jurisprudence ${ }^{5}$, comme en témoigne sa nouvelle appellation.

Nous avons cherché à déterminer si la position des légistes, à l'effet que le droit nouveau se limite généralement à des modifications mineures et maintient le statu quo sur le fond ${ }^{6}$, se vérifie également au sujet de l'état d'esprit du débiteur et de la partie contractant avec lui. Il nous a semblé intéressant de nous pencher sur cet aspect de l'action en inopposabilité, en nous attardant plus spécifiquement à l'acte conclu à titre onéreux ${ }^{7}$. Est-il possible que le législateur ait malgré tout bouleversé le régime élaboré sous l'ancien Code? Pour répondre à cette question, il convient de distinguer l'état d'esprit requis du débiteur (I) et du tiers avec lequel celui-ci contracte (II).

\section{L'intention frauduleuse du débiteur}

L'intention frauduleuse du débiteur se trouve au coeur de l'action en inopposabilité. L'étude du droit antérieur révèle un régime intéressant et généralement bien équilibré (A). Il en va autrement du droit nouveau où, malgré des efforts louables du législateur, cet équilibre semble mis en péril (B).

4. Tout comme c'était le cas avant la réforme (art. 1039 C.c.B.-C.), la créance invoquée par le demandeur doit être antérieure à l'acte attaqué, bien que le législateur prévoie désormais une exception à ce principe (art. 1634 al. 2 C.c.Q.). Là encore, il ne s'agit en réalité que d'une codification de règles suggérées par la doctrine et adoptées en jurisprudence; à cet effet, voir notamment: P.-B. Mignault, Le droit civil canadien, t. 5, Montréal, Théorêt, 1901, p. 294; Trudel, supra, note 1, p. 498.

La réforme vient par ailleurs trancher la question liée au caractère certain, liquide et exigible de la créance (art. 1634 al. 1 C.c.Q.), adoptant à cet égard une règle identique à celle introduite dans l'action oblique (art. 1627 al. 1 et 1628 C.c.Q.). Sur la controverse à cet égard, dans le droit antérieur, voir notamment: Banque Nationale de Paris c. Mandelker, [1985] C.S. 280, p. 283-285; Daigle c. Rousseau, [1987] R.J.Q. 397 (C.S.), p. 401-402.

5. Ministère de la Justice, Commentaires du ministre de la Justice, t. I, Québec, Publications du Québec, 1993, p. 1012 (sous l'art. 1631).

6. Commentaires du ministre de la Justice, t. I, ibid., p. 1011-1012 (sous les art. 1631-1632 C.c.Q.).

7. Il est vrai que, dans le droit antérieur, l'intention frauduleuse du débiteur est requise pour l'acte à titre gratuit comme pour celui conclu à titre onéreux (art. 1033 C.c.B.-C.). Le régime applicable varie pourtant de façon notable selon la nature du contrat concerné.

Ainsi, bien que le mot «réputé» soit utilisé dans les deux articles énonçant les cas de présomption d'intention frauduleuse, celle relative au contrat à titre gratuit est considérée comme irréfragable, contrairement à ce qui prévaut en matière de contrat à titre onéreux: J. Pineau et D. Burman, Théorie des obligations, $2^{\mathrm{e}}$ éd., Montréal, Éditions Thémis, 1988, ${ }^{\circ}$ 396. De plus, l'état d'esprit de la personne contractant avec le débiteur n'est pas considérée dans l'acte à titre gratuit, puisque l'art. 1038 C.c.B.-C. se limite aux contrats à titre onéreux. Ce régime distinct pour l'acte à titre gratuit s'explique aisément du fait de l'absence de toute contrepartie par le cocontractant. C'est en raison de cette réalité économique toute particulière qu'il convient de limiter notre propos à l'acte à titre onéreux. 


\section{A. Le droit antérieur}

Déjà, dans le droit romain, la preuve d'une intention frauduleuse était requise en matière paulienne ${ }^{8}$. Cette condition a paru essentielle aux codificateurs français, puisque le Code énonce que les créanciers peuvent «attaquer les actes faits par leur débiteur en fraude de leurs droits» (art. 1167 C.c. fr.). L'élément de fraude a également obtenu la faveur des codificateurs québécois de 1866, bien que la formulation diffère quelque peu du modèle français: «Un contrat ne peut être annulé à moins qu'il ne soit fait avec l'intention dé frauder, et qu'il n'ait l'effet de nuire au créancier» (art. 1133 C.c.B.-C.).

Que doit-on entendre par «intention frauduleuse»? Malgré la fluidité de cette notion, doctrine et jurisprudence s'entendent généralement pour y voir, en matière paulienne, la connaissance par un débiteur du préjudice causé à ses créanciers du fait de ses agissements?

L'intention frauduleuse se situe au-delà de la simple négligence ${ }^{10}$. Le débiteur doit avoir connaissance ou conscience de l'effet préjudiciable susceptible de résulter de l'acte ${ }^{11}$. Si une telle connaissance est requise pour conclure à l'existence d'une intention frauduleuse, elle est également suffisante. Il faut en effet se garder de distinguer selon l'importance des manoeuvres entreprises par le débiteur en vue de nuire à ses créanciers. La fraude «passive» du débiteur opportuniste, tout comme la fraude «active» du débiteur malhonnête, sont également sanctionnées. En un mot, l'intention frauduleuse est synonyme, en matière paulienne, de mauvaise foi du débiteur à l'endroit de ses créanciers ${ }^{12}$. Il est indifférent que le débiteur, en agissant de la sorte, n'ait pas songé à frauder un créancier en particulier ${ }^{13}$.

Le fardeau de prouver l'intention frauduleuse du débiteur incombe au créancier qui cherche à obtenir l'inopposabilité de l'acte attaqué. La preuve directe d'une telle intention demeure difficile, en raison même du caractère occulte de la fraude. Aussi, dans plusieurs cas, le créancier-demandeur devra,

8. C. Accarias, Précis de droit romain, t. II, $4^{\mathrm{e} e}$ éd., Paris, F. Pichon, $1891, \mathrm{n}^{\circ} 851$.

9. $\quad$ En ce sens, l'intention frauduleuse présente des liens indéniables avec l'autre condition charnière de l'action en inopposabilité, soit l'existence d'un préjudice effectif chez le créancier-demandeur.

10. Ceci contraste avec l'action oblique, pour laquelle la simple négligence du débiteur suffit.

11. V. Marcadé, Explication théorique et pratique du Code Napoléon, $5^{\mathrm{e}}$ éd., t. 4, Paris, Cotillon, 1859, p. 403.

12. J.-L. Baudouin, Les obligations, $3^{\mathrm{e}}$ éd., Cowansville, Éditions Yvon Blais, 1989, $\mathrm{n}^{\circ}$ 595; Pineau et Burman, supra, note 7, n ${ }^{\circ} 395$; G.A. Rosenberg, «The Notion of Good Faith in the Civil Law of Quebec», (1960) 7 R.D. McGill 1, p. 18. Voir aussi: Duchesne c. Labbé, [1973] C.A. 1002, p. 1005; Biron c. Poirier, [1978] C.S. 231, p. 238; Royal Bank of Canada c. Rousseau, [1977] C.S. 1090, p. 1093. Trudel, supra, note 1, p. 458.

13. Toullier, supra, note $2, \mathrm{n}^{\circ} 349$. 
selon le droit commun de la preuve, établir cette intention à l'aide de présomptions de faits (art. 1242 C.c.B. - C. $)^{14}$.

Malgré tout, la tâche du créancier peut s'avérer difficile. C'est pourquoi les codificateurs de 1866 ont prévu différents types de présomptions légales destinées à faciliter la preuve de l'intention frauduleuse du débiteur ${ }^{15}$.

Dans le cas d'un acte à titre onéreux, deux éléments doivent être réunis par le créancier pour profiter d'une présomption légale d'intention frauduleuse: l'insolvabilité du débiteur au moment de l'acte et la connaissance par le tiers de cette insolvabilité (art. 1035 C.c.B.-C.). Pourquoi présume-t-on qu'un contrat est fait avec l'intention de frauder simplement parce que l'une des parties au contrat connaît l'insolvabilité de l'autre? Tout comme le débiteur insolvable est en mesure d'anticiper les conséquences de l'acte juridique sur son patrimoine insuffisant, tout autant le cocontractant informé de cette insolvabilité peut apprécier les conséquences de l'acte intervenu. En d'autres mots, la conclusion d'un acte, alors que l'insolvabilité de l'une des parties est connue de l'autre, laisse croire à l'existence d'un «climat», d'une ambiance propice à la fraude des droits des créanciers de l'insolvable.

Pourtant, une telle intention n'existe pas toujours chez le débiteur insolvable qui contracte avec des tiers informés de son insolvabilité. Ce débiteur peut contracter alors que son insolvabilité est connue, tout en cherchant à conclure un marché régulier ${ }^{16}$. Il n'est donc pas surprenant que la jurisprudence ait reconnu, de concert avec la doctrine, le caractère réfragable de la présomption d'intention frauduleuse malgré l'utilisation du mot «réputé» à l'art. 1035 C.c.B. - C. ${ }^{17}$.

14. Biron c. Poirier, supra, note 12, p. 236-237. Trudel, supra, note 1, p. 483; Baudouin, $3^{\mathrm{e}}$ éd., supra, note $12, \mathrm{n}^{\circ} 595$.

15. Les commentaires relatifs à la codification de 1866 nous enseignent que ces présomptions trouvent leur source en droit romain, lequel était invoqué par la jurisprudence et la doctrine françaises à défaut de présomptions légales dans le Code civil français: Code civil du BasCanada. Premier, second et troisième rapports, Québec, George E. Desbarats, 1865, p. 1517.

Malgré cette intention avouée de modeler ce régime sur les principes reconnus en droit français à l'époque de la codification, force est de constater que ce dernier a connu des développements jurisprudentiels et doctrinaux importants. Du même coup, l'approche comparative doit être empreinte de prudence. Sur les règles applicables à cet égard en droit français actuel, voir notamment: H., L. et J. Mazeaud, Leçons de droit civil, t. 2, vol. 1, Obligations. Théorie générale, $8^{\mathrm{e}}$ éd. par F. Chabas, Paris, Montchrestien, 1991, n ${ }^{\text {os }} 993-$ 997.

16. Sur la difficulté de faire une telle preuve, voir néanmoins: Baudouin, $3^{\mathrm{e}}$ éd., supra, note 12, $\mathrm{n}^{\circ} 595$.

17. In re Callway Sash and Door Inc.: Perras c. Banque provinciale du Canada, [1963] B.R. 919, p. 923; In re Gérard Nolin Ltée: Banque Canadienne Nationale c. Bellavance, [1979] C.A. 168, inf. (sub nom. In re Gérard Nolin Ltée: Bellavance c. Trust Général du Canada), 
Deux traits majeurs du droit antérieur peuvent être relevés au sujet de l'intention frauduleuse du débiteur.

D'une part, le Code marque clairement la distinction entre cette condition de l'action paulienne (art. 1033 C.c.B.-C.) et la présomption qui en facilite la preuve (art. 1035 C.c.B.-C.); ainsi, les éléments constitutifs de la présomption ne représentent pas des conditions sine qua non du recours ${ }^{18}$. Il est donc possible d'imaginer l'existence d'une intention frauduleuse chez un débiteur solvable $^{19}$, dont l'insolvabilité ne peut être prouvée ${ }^{20}$, ou encore dont l'état d'insolvabilité est inconnu du tiers qui contracte avec lui ${ }^{21}$. Le créancier peut toujours se prévaloir de l'action en inopposabilité, mais il lui incombe alors de démontrer l'intention frauduleuse du débiteur par preuve directe ou par présomption de faits.

D'autre part, dans le contexte des présomptions légales, le droit maintient un équilibre entre les intérêts du créancier et ceux du débiteur: le premier profite d'un renversement du fardeau de preuve à l'égard d'un élément difficile à cerner, alors que le second peut démontrer une réalité différente de ce que la loi laisse présumer. la réforme.

Reste à déterminer si ces traits essentiels ont été repris dans le cadre de

\section{B. Le droit nouveau}

Le législateur québécois réaffirme, dans le Code civil du Québec, le rôle central de l'intention frauduleuse du débiteur dans l'action en inopposabilité. Cet aspect de la réforme, qui semble à première vue aller de soi, mérite néanmoins d'être signalé. En effet, l'Office de révision du Code civil proposait d'éliminer cette condition, du moins dans les cas où le débiteur était ou se rendait

C.S. Québec, 200-11-000302-748, 26 mars 1975, M. le juge Jacques, [1975] C.S. 770 (som.). Il en va autrement dans le droit nouveau; à ce sujet, voir l'art. 2847 C.c.Q. et cidessous, partie I.B.

18. Trudel, supra, note 1, p. 502; P.W. Morin, «Le recours paulien en matière de faillite et en matière civile», (1973) 33 R. du B. 41, p. 59.

19. Il en va ainsi du débiteur qui transforme ses immeubles en effets négociables en vue de flouer ses créanciers: Langelier, t. 3, supra, note 2, p. 437. Par exemple, Duchesne c. Labbé, supra, note 12; Biron c. Poirier, supra, note 12; Droit de la famille - 1824, C.S. SaintFrançois, 450-05-000994-901, 17 mai 1993, M. le juge Péloquin, J.E. 93-1269 (en appel: 500-09-001157-932).

20. Voir, par exemple, Bouchard c. Parent, C.A. Montréal, 500-09-001357-847, 5 mai 1988, M. le juge LeBel, J.E. 88-780,.

21. Voir, par exemple, Spenard c. Lapointe, C.S. Drummond, 405-05-000060-848, 11 août 1987, M. le juge Frappier, J.E. 87-1258, p. 15-16. 
insolvable ${ }^{22}$. Dès l'avant-projet de loi sur les obligations (art. 1685) et jusqu'à l'adoption du projet de loi 125 (art. 1629, devenu l'art. 1631 C.c.Q.), le législateur a privilégié le maintien de cette exigence ${ }^{23}$.

Certains points positifs de la réforme méritent d'être soulignés.

Ainsi, dans le cadre de la théorie générale des obligations, on reconnaît désormais explicitement le devoir de bonne foi des parties dans l'exécution des obligations (art. 1375 C.c.Q. $)^{24}$. Ce principe d'application générale constitue une toile de fond pour le recours en inopposabilité, lequel intervient lorsqu'un acte juridique met en péril le patrimoine d'un débiteur, gage commun de ses créanciers (art. 2644-2645 C.c.Q.).

Par ailleurs, la réforme reprend la distinction entre le droit du créancier de réclamer l'inopposabilité du contrat frauduleux (art. 1631 C.c.Q.), d'une part, et les présomptions destinées à en faciliter la preuve (art. 1632 et 1633 C.c.Q.), d'autre part.

Au sujet des présomptions, le Code civil du Québec conserve l'élément de connaissance du tiers au nombre des faits objectifs permettant l'établissement d'une présomption légale d'intention frauduleuse du débiteur. L'hypothèse envisagée à l'art. 1035 C.c.B.-C. - l'acte conclu par le débiteur insolvable - est conservée à l'art. 1632 C.c.Q. Le nouveau Code précise désormais que l'insolvabilité créée par l'acte attaqué est également visée par cette présomption. Il ne s'agit là, en fait, que d'une extension logique du principe général. Là où le droit nouveau innove de façon plus significative, c'est en incluant dans le domaine de la présomption l'hypothèse du débiteur qui cherche à se rendre

22. Le Projet de Code civil prévoyait également le cas où le débiteur cherche à se rendre insolvable, une situation qui, nous le verrons plus loin, correspond généralement à un comportement de mauvaise foi constitutif de fraude. Sur les modifications proposées, voir : Office de révision du Code civil, Rapport sur le code civil, vol. I, Projet de code civil, Québec, Éditeur officiel du Québec, 1977, art. 197; ibid., vol. II, Commentaires, t. 2, p. 658659.

23. Les légistes répondent implicitement aux réserves exprimées par l'ORCC en mentionnant la largesse de la jurisprudence et de la doctrine quant à la preuve de l'intention frauduleuse: Commentaires du ministre de la Justice, t. I, supra, note 5, p. 1011-1012 (sous l'art. 1631 C.c.Q.).

24. Cette idée était déjà défendue par Toullier, selon qui la bonne foi exigée dans les conventions ne se borne pas au cocontractant mais est due à tous ceux que peut intéresser ce qui se passe entre eux: Toullier, t. 6 , supra, note $2, \mathrm{n}^{\circ} 342$. Ce principe est exprimé en termes plus larges encore au nouvel art. 1375 C.c.Q., lequel impose un devoir de bonne foi dans l'exécution des obligations quelles qu'en soient la source - ce qui vise notamment les agissements du débiteur à l'égard de son patrimoine. 
insolvable, sans pour autant le devenir par cet acte ${ }^{24 a}$. L'ajout de cette troisième hypothèse n'est pourtant pas de nature à bouleverser le fonctionnement de l'action en inopposabilité. Sans mettre en doute la pertinence d'un tel ajout, qu'il suffise de mentionner que, contrairement à l'insolvabilité elle-même, la volonté de devenir insolvable constitue un fait subjectif. Un créancier qui réussit à faire la preuve d'une telle volonté chez le débiteur peut, la plupart du temps, démontrer une intention frauduleuse par preuve directe. Le bénéfice d'une présomption légale s'avère alors superflue ou, à tout le moins, d'une utilité limitée.

Les premiers commentateurs s'entendent pour regretter un aspect de la réforme, soit la possible mutation de la présomption d'intention frauduleuse du débiteur en présomption irréfragable ${ }^{25}$. En effet, selon le nouvel article $2847 \mathrm{al}$. 2 C.c.Q., la présomption concernant des faits «réputés» est absolue et ne permet aucune preuve contraire - ce que semble exprimer l'art. 1632 C.c.Q.

S'agit-il là d'une modification intentionnelle ou d'un simple incident de parcours? Divers indices tendent vers la seconde interprétation ${ }^{26}$.

Ainsi, il ne serait pas surprenant que le législateur ait transposé le texte de l'article 1035 C.c.B.-C. sans s'arrêter au sens accordé au mot «réputé» dans le droit nouveau. En effet, ce mot était auparavant utilisé indistinctement pour désigner une présomption simple ou irréfragable, et la jurisprudence avait jugé bon d'y voir une présomption réfragable. Or, la réforme a amené, au chapitre de la preuve, une utilisation plus rationnelle des mots «réputé» et «présumé»: le

24a Il faut noter que dans l'hypothèse où le débiteur cherche à créer l'illusion de l'insolvabilité par des actes fictifs ou déguisés, les dispositions relatives à la simulation sont à la disposition du créancier (art. 1451 C.c.Q.). Sur le domaine respectif des deux recours, voir notamment: Trudel, supra, note 1, p. 439 et suiv. La distinction entre l'action en inopposabilité et le recours en déclaration de simulation est toutefois passée sous silence par les légistes: Commentaires du ministre de la Justice, t. I, supra, note 5, p. 1012 (sous l'art. 1631 C.c.Q.).

25. Sur cette question, voir généralement: J. Pineau, «Théorie des obligations» dans Barreau du Québec et Chambre des notaires du Québec, La réforme du Code civil. Obligations et contrats nommés, Québec, Presses de l'Université Laval, 1993, 9, nº 180; L. Ducharme, «Le nouveau droit de la preuve en matière civile selon le Code civil du Québec», (1992) 23 R.G.D. 5, p. 45; A.-F. Bisson, «Nouveau Code civil et jalons pour l'interprétation: traditions et transitions», (1992) 23 R.D.U.S., p. 18.

Déjà sous l'art. 1035 C.c.B.-C., les professeurs Pineau et Burman relevaient l'injustice que pourrait causer une présomption irréfragable d'intention frauduleuse dans les contrats à titre onéreux: Pineau et Burman, supra, note 7, n 396.

26. Les commentaires des légistes apportent peu de précisions sur ce point. Ainsi, on peut y lire que les dispositions du droit antérieur ont été reprises mais que la connaissance de l'insolvabilité constitue désormais un obstacle à la bonne foi: Commentaire du ministre de la Justice, t. I, supra, note 5, p. 1012 (sous l'art. 1632 C.c.Q.). Il est toutefois difficile de déceler si cet obstacle se rapporte à la preuve de bonne foi du débiteur, du tiers cocontractant ou encore de l'un et l'autre à la fois. Á tout événement, les motifs d'un tel resserrement n'y sont pas exposés. 
premier réfère à une présomption irréfragable, alors que le second laisse place à une preuve contraire (art. 2847 al. 2 C.c.Q.). Une transcription mécanique de la terminologie utilisée à l'article 1035 C.c.B.-C. aurait ainsi amené la transformation d'une présomption simple en présomption irréfragable ${ }^{27}$.

L'hypothèse d'une erreur de rédaction est d'autant plus plausible que les projets de réforme ont mis de l'avant, pour un temps du moins, la possibilité d'un recours en inopposabilité dépouillé de la condition d'intention frauduleuse et des présomptions légales élaborées dans le droit antérieur ${ }^{28}$. En revenant à ce régime initial, les codificateurs ont peut-être perdu de vue l'importance de reconnaître le caractère réfragable de la présomption découlant de l'article 1035 C.c.B.-C.

En supposant qu'il s'agit effectivement d'un concours de circonstances, serait-il néanmoins souhaitable de conserver une telle modification afin d'assurer une meilleure protection des intérêts des créanciers d'un insolvable? Nous ne le croyons pas. Il est vrai que les créanciers d'un insolvable se trouvent dans une situation précaire. Toutefois, le droit a tout intérêt à rechercher un équilibre entre les droits de ces derniers et la stabilité des transactions effectuées par le débiteur avec des tiers ${ }^{29}$. Or, la preuve de bonne foi du débiteur - c'est-à-dire de son absence d'intention frauduleuse - constituait une justification fort adéquate pour limiter la protection des droits du créancier.

Il est permis d'imaginer certaines conséquences susceptibles de résulter d'une protection aussi large des intérêts des créanciers de l'insolvable. Ainsi, certaines personnes éprouveront-elles une plus grande hésitation lorsque viendra le temps de contracter avec une partie qu'elles savent être insolvable, et ce, même si l'honnêteté de cette dernière ne fait pas de doute. Qu'il suffise de penser au banquier et au fournisseur qui, acceptant de conclure un marché avec un insolvable, peuvent aussi contribuer au redressement de sa situation financière. Par ailleurs, le débiteur insolvable sera peut-être, à l'occasion, tenté de camoufler son état financier de façon à sécuriser son cocontractant et, du même coup, éviter l'application d'une présomption irréfragable d'intention frauduleuse ${ }^{29 a}$.

27. En ce sens, voir également : Pineau, supra, note 25, $\mathrm{n}^{\circ}$ 180; Ducharme, supra, note 25, p. 45; Bisson, supra, note 25, p. 18.

28. Voir: Rapport sur le code civil, supra, note 22; voir aussi ci-dessus, partie I.A.

29. Cette réalité est reconnue dans le droit de la faillite, où le failli peut démontrer que la transaction a été effectuée dans le cours normal des affaires, la présomption de préférence frauduleuse étant réfragable: Loi sur la faillite, L.R.C. (1985), c. B-3, art. 95 par. 2 [anciennement Loi sur la faillite, S.R.C. 1970, c. B-3, art. 73]; Morin, supra, note 18, p. 4647.

29a Au sujet de telles omissions dans le cadre de rapport contractuels, il convient de souligner le nouvel art. 2159 al. 2 C.c.Q. qui, en matière de mandat, impose au mandataire le devoir de faire connaître l'état d'insolvabilité de son mandant à peine d'engager sa responsabilité 
(1992) 23 R.D.U.S.

La bonne foi des parties au contrat à titre onéreux dans l'action en inopposabilité :

Dans l'affaire In re Gérard Nolin Ltée: Banque canadienne nationale c. Bellavance, la Cour d'appel réfutait la théorie selon laquelle l'art. 1035 C.c.B.-C. créait une présomption juris et de jure. Les propos du juge Bernier conservent toute leur pertinence dans le contexte de l'art. 1632 C.c.Q.:

Une telle théorie aurait en pratique pour effet de réduire le débiteur insolvable à l'état d'incapable, ce que le Législateur n'a certes pas voulu. En effet, tel débiteur se verrait dès lors incapable de passer quelque contrat à titre onéreux que ce soit, qui ne serait pas, de plein droit, sujet à annulation à l'instigation de l'un quelconque de ses créanciers ou du syndic en cas de faillite; et ce, nonobstant non seulement l'absence totale de préjudice pour les créanciers, mais même s'il devait en résulter un bénéfice, pas même en vue de se sortir de son état d'insolvabilité, à moins de ne traiter qu'avec des personnes qui ignorent son insolvabilité et à qui il prend garde de le révéler. Cette proposition, à mon avis, répugne ${ }^{30}$.

Ces considérations nous amènent à conclure à la nécessité de rétablir le régime prévalant dans le droit antérieur, soit le caractère réfragable de la présomption d'intention frauduleuse. Mais toute nécessaire qu'elle soit, cette modification serait malgré tout insuffisante. Aux difficultés relatives à la présomption d'intention frauduleuse s'ajoutent, en effet, des interrogations quant à la défense de bonne foi du tiers.

\section{La mauvaise foi du tiers}

L'action en inopposabilité, par définition, affecte un tiers ayant contracté avec le débiteur de la partie demanderesse. La mécanique du recours donne-telle un droit de parole à ce tiers pour lui permettre d'éviter que l'acte conclu ne soit déclaré inopposable? Le droit antérieur le permettait à certaines conditions (A), ce qui ne semble plus être le cas dans le droit nouveau (B).

\section{A. Le droit antérieur}

L'art. 1038 C.c.B.-C. offre au tiers, confronté à l'éventuelle inopposabilité d'un acte à titre onéreux conclu au préjudice d'autres créanciers de son cocontractant, la possibilité de conserver les effets de cet acte en démontrant sa

personnelle - ce qui vise notamment l'administrateur d'une personne morale (art. 321 C.c. Q.). Qu'il divulgue ou non l'état d'insolvabilité de son mandant, le mandataire subira des conséquences particulièrement défavorables.

30.

Supra, note 17, p. 175-176. 
bonne foi dans le cadre de la transaction, et ce, malgré la preuve de l'intention de frauder du débiteur ${ }^{31}$.

Comment se définit la bonne ou mauvaise foi du tiers? Il est permis de tracer ici un parallèle avec la notion d'intention frauduleuse telle que nous l'avons envisagée plus haut: un tiers agit de bonne foi lorsqu'il conclut un acte tout en ignorant le préjudice qui en résulte pour les créanciers de son cocontractant ou, formule voisine, qu'il ignore les circonstances susceptibles d'entraîner l'inopposabilité du contrat ${ }^{32}$.

Ici encore, il faut noter que la notion de mauvaise foi dépasse la simple négligence. On ne saurait donc reprocher au tiers de n'avoir pas entrepris de démarches permettant de connaître en détail la condition patrimoniale de son vis-à-vis au moment de la transaction ${ }^{33}$.

Par contre, la mauvaise foi ne nécessite pas une participation active du tiers à la création d'un préjudice pour les créanciers de son cocontractant. Un tiers peut être considéré de mauvaise foi s'il accepte de passer un contrat tout en soupçonnant les difficultés financières de son cocontractant ou encore les démêlés de ce dernier avec ses créanciers. La passivité du tiers ne permet pas de l'innocenter. Comme le précisait le juge Gagnon dans l'affaire Duchesne c. Labbé:

Je laisse les auteurs français à leur querelle sur la question de savoir si telle connaissance suffit ou si la participation de celui qui contracte avec le débiteur doit aller jusqu'à la complicité. L'article 1038 C.C. parle de bonne foi et non de complicité et l'autorité de ce texte me

31. Limite importante du droit d'un créancier lésé d'obtenir l'inopposabilité d'un acte, ce moyen de défense est cependant restreint aux actes à titre onéreux. L'art. 1038 C.c.B.-C. exclut clairement le cas de l'acte à titre gratuit, ce qui s'explique facilement du fait que le tiers n'a alors donné aucune contrepartie pour la prestation du débiteur. Il répugne à la logique comme à la justice que le tiers conserve une libéralité au détriment d'un créancier fraudé. De plus, la jurisprudence confirme que l'art. 1038 C.c.B.-C. ne peut être invoqué par le tiers lorsque le créancier attaque un paiement effectué par son débiteur plutôt qu'un contrat conclu par ce dernier: In re Société coopérative agricole régionale de La Tuque: Benoît Lafrance Inc. c. Verroeulst, [1974] C.A. 435, p. 438.

32. Labbé c. Nolin, [1972] C.S. 418, p. 422, conf. (sub nom. Duchesne c. Labbé), supra, note 12; Royal Bank of Canada c. Rousseau, supra, note 12, p. 1093; In re Gérard Nolin Ltée: Bellavance c. Trust Général du Canada, supra, note 17, p. 35-36, inf. sur un autre point (sub nom. In re Gérard Nolin Ltée: Banque Canadienne Nationale c. Bellavance), supra, note 17; Spenard c. Lapointe, supra, note 21, p. 16-18; Daigle c. Rousseau, supra, note 4, p. 402. Voir aussi: Gagnon c. St-Denis, C.S. Longueuil, 505-05-000646-908, 15 octobre 1992, M. le juge Chabot, J.E. 92-1757, p. 8-10.

33. Voir: M. Tancelin, Des obligations. Contrats et responsabilité, $4^{\mathrm{e}}$ éd., Montréal, Wilson et Lafleur, 1988, n 665. 
paraît suffisant pour écarter la complicité comme condition de l'action paulienne ${ }^{34}$.

Il arrive que la mauvaise foi du tiers soit présentée comme l'une des conditions de l'action paulienne ${ }^{35}$. À notre avis, cette affirmation mérite d'être nuancée. En effet, le créancier a tout intérêt à démontrer, dans le cadre de son recours, la mauvaise foi du tiers qui a contracté à titre onéreux avec son débiteur. Toutefois, au plan théorique, la bonne foi du tiers constitue plutôt un moyen de défense ${ }^{36}$. Le fardeau incombe alors au tiers de prouver sa bonne foi, bien que le créancier-demandeur puisse, par anticipation, chercher à démontrer le contraire. C'est l'existence d'une intention frauduleuse de la part du débiteur qui explique la nécessité d'une preuve de bonne foi par le tiers. Par mesure de prudence, ce dernier tentera généralement de plaider sur les deux plans: son cocontractant, le débiteur, n'avait pas d'intention frauduleuse, alors que luimême était de bonne foi. À défaut, pour les deux parties au contrat, de faire reconnaître l'absence d'intention frauduleuse du débiteur, le moyen de défense propre au tiers reste le seul moyen d'éviter l'inopposabilité de l'acte conclu. À cet égard, il est indifférent que l'intention frauduleuse du débiteur ait été prouvée conformément au droit commun de la preuve ou qu'elle découle de la présomption légale de l'art. 1035 C.c.B.-C.

L'art. 1038 C.c.B.-C. apparaît comme une atténuation au principe selon lequel la bonne foi d'une personne se présume (art. 2202 C.c.B.-C.). Dès lors qu'un contrat est conclu par le débiteur en fraude des droits de ses créanciers, il est logique de présumer, jusqu'à preuve du contraire, que les deux parties à cet acte sont également de mauvaise foi ${ }^{37}$.

Le moyen de défense offert par l'article 1038 C.c.B.-C. n'est pas simplement théorique, même dans les cas où le tiers avait connaissance de l'insolvabilité du débiteur. Ce tiers peut connaître l'insolvabilité de son

34. Supra, note 12, p. 1010. Dans le même sens, voir: Trudel, supra, note 1, p. 486-487.

35. Notamment Duchesne c. Labbé, ibid., p. 1005. Voir aussi: Meunerie Camirand Inc. c. Banque Nationale du Canada, C.A. Montréal, 500-09-000212-860, 9 mars 1993, J.E. 93583.

36. Maurice D'Arcy et associés Inc. c. Construction St-Viateur Inc., C.S. Montréal, 500-05003246-822, 18 juin 1982, M. le juge Benoît, J.E. 82-775 (C.S.), p. 10-11. Trudel, supra, note 1, p. 494. Voir aussi: In re Koko Enterprises Ltd.: Waxman c. Toronto Dominion Bank, C.S. Montréal, 500-11-000568-754, 18 janv. 1979, M. le juge Lévesque, J.E. 79-159, p. 15; Jenner c. Seligman, C.S. Montréal, 500-05-009597-749, 8 septembre 1980, M. le juge Lévesque, J.E. 80-888 (C.S.), p. 19; Daigle c. Rousseau, supra, note 4, p. 402; In re Bolduc et associés Inc.: Swidler c. Laurent Gagnon Inc., [1978] C.S. 538, p. 541-542.

37. Jenner c. Seligman, ibid., p. 19: «En matière de droit paulien, la bonne foi ne se présume pas et c'était aux défendeurs à se décharger du fardeau de la preuve et à venir établir qu'il y avait des considérations valables équivalentes à la valeur du terrain cédé.» Morin, supra, note 18 , p. 60. Voir aussi: Trudel, ibid., p. 494; Baudouin, $3^{\mathrm{e}}$ éd., supra, note 12, no 597. 
cocontractant et, malgré tout, chercher à conclure un marché régulier ${ }^{38}$. La preuve du tiers sera plus ou moins facile selon les circonstances entourant la conclusion de l'acte ${ }^{39}$. Ainsi, lorsque le tiers est un proche du débiteur (conjoint, parent, administrateur ou actionnaire d'une personne morale, etc.), il lui sera plus difficile de démontrer qu'il ignorait les circonstances permettant d'attaquer l'acte $^{40}$ que dans le cas d'un fournisseur peu au fait des intentions de son cocontractant à l'égard de ses autres créanciers.

Tout comme c'était le cas au sujet de l'intention frauduleuse du débiteur, les règles relatives à la bonne ou mauvaise foi du tiers tendent au maintien d'un équilibre entre les intérêts respectifs du créancier (victime d'une atteinte au patrimoine de son débiteur) et du tiers (victime de l'instabilité des rapports contractuels). Le tiers qui connaît bien la situation ne peut s'en prendre qu'à luimême. Par contre, s'agissant d'un tiers innocent, la loi favorise ce dernier au détriment du créancier lésé ${ }^{41}$.

Le tiers, amené à son insu par un cocontractant de mauvaise foi à conclure un acte préjudiciable aux créanciers de ce dernier, pourrait être dans une position beaucoup moins avantageuse dans le droit nouveau.

\section{B. Le droit nouveau}

Dans leurs commentaires, les légistes mentionnent que l'art. 1632 C.c.Q. «regroupe, dans une formulation plus simple et plus précise, l'essentiel des dispositions des articles 1035, 1036 et 1038 C.C.B.C. [...] $\gg^{42}$. Pourtant, on n'y trouve aucun élément permettant d'identifier un quelconque moyen de défense personnel au tiers de bonne foi. Qui plus est, cet article concerne uniquement l'hypothèse où la loi présume l'intention frauduleuse du débiteur, alors que l'on a vu précédemment que l'art. 1038 C.c.B.-C. permettait au tiers d'invoquer sa bonne foi peu importe le mode de preuve par lequel l'intention frauduleuse du débiteur était établie ${ }^{43}$. Les légistes sont laconiques sur ce point:

38. Trudel, ibid., p. 493; Baudouin, ibid., n ${ }^{\circ} 597$.

39. Sur l'appréciation de la bonne foi du tiers en application de l'art. 1038 C.c.B.-C., voir : Morin, supra, note 18, p. 60-61; Rosenberg, supra, note 12, p. 16-17. Voir aussi: Labbé c. Nolin, supra, note 32, p. 423-425, conf. (sub nom. Duchesne c. Labbé), supra, note 12, p. 1010-1011.

40. Par exemple, Royal Bank of Canada c. Rousseau, [1977] C.S. 1090, p. 1093-1094; Biron c. Poirier, supra, note 12, p. 237; Daigle c. Rousseau, supra, note 4, p. 402; Droit de la famille - 1824, supra, note 19; Sous-ministre du Revenu du Québec c. Assurances Arbour Inc., C.Q. Québec, 200-02-009824-873, 30 mars 1990, M. le juge Beaulieu, D.F.Q.E. 90F52; Lapointe c. Goyer, [1986] R.J.Q. 257 (C.S.).

41. Toullier, supra, note $2, \mathrm{n}^{\circ} 352$.

42. $\quad$ Commentaires du ministre de la Justice, t. I, supra, note 5, p. 1012 (sous l'art. 1632) [nos italiques].

43. Voir: Trudel, supra, note 1, p. 487. 
Comme dans le passé, il [l'article 1632] préserve les droits du tiers de bonne foi qui a fourni valeur en contrepartie du contrat ou du paiement. Cependant, les exigences de la bonne foi sont resserrées, puisque la connaissance de l'état d'insolvabilité, atteinte ou recherchée, du débiteur constituera désormais un obstacle à la bonne foi ${ }^{44}$.

Une lecture des articles 1631 et 1632 C.c.Q. nous porte à croire que la substance de l'art. 1038 C.c.B.-C. a été purement et simplement omise dans le cadre de la réforme. Quant aux motifs qui animaient le législateur, il est fort à parier qu'il s'agit, là encore, d'une modification fortuite.

Ainsi, rien n'indique que le législateur ait cherché à s'inspirer du régime applicable en matière de contrat à titre gratuit ${ }^{45}$ ou de paiement préférentiel ${ }^{46}$ pour priver le tiers d'un moyen de défense personnel fondé sur sa bonne foi.

Cette approche du législateur pourrait résulter d'une certaine confusion entre l'intention frauduleuse du débiteur et la mauvaise foi du tiers. Il faut concéder que l'une et l'autre sont très similaires au plan conceptuel, reposant sur la connaissance du caractère préjudiciable de l'acte attaqué pour les créanciers de l'un des cocontractants. Pourtant, elles sont distinctes dans les faits puisque l'on peut fort bien imaginer la situation où un tiers innocent transige avec un débiteur mû par une intention frauduleuse. C'est précisément l'hypothèse qu'envisageait l'art. 1038 C.c.B.-C.

44. Commentaires du ministre de la justice, t. I, supra, note 5, p. 1012 (sous l'art. 1632). Le professeur Baudouin, dans la plus récente édition de son ouvrage, semble néanmoins reconnaître la possibilité pour le tiers de démontrer sa bonne foi: Baudouin, $4^{\mathrm{e}}$ éd., supra, note $3, n^{\circ} 684$

45. La nature particulière des rapports économiques générés par un acte à titre gratuit, nous l'avons mentionné plus tôt, rend difficile tout parallèle quant à la position du tiers bénéficiant de la prestation offerte par le débiteur. Si le législateur avait souhaité imposer un régime identique dans les contrats à titre gratuit et onéreux, on peut imaginer qu'il l'aurait fait de façon plus explicite.

46. Dans le droit de la faillite, la seule bonne foi du tiers est insuffisante pour éviter l'application du recours pour paiement préférentiel: Hudson c. Bellanack, [1976] 2 R.C.S. 168. Il faut nécessairement démontrer que le débiteur n'agissait pas avec une intention frauduleuse, notamment en prouvant qu'il s'agissait d'un paiement effectué par le débiteur dans le cours normal de ses affaires: ce n'est donc pas la bonne foi du tiers qui importe, mais plutôt l'absence d'intention de frauder de la personne insolvable. L'intention de préférer n'a donc pas à être commune au cocontractant. À ce sujet, voir notamment: P. Côté, «Le traitement préférentiel accordé à un créancier au cours de la période suspecte», (1967) 2 R.J.T. 287, p. 306-308; Morin, supra, note 18, p. 47 et suiv.; Tancelin, supra, note 33, n ${ }^{\circ} 666$ et n. 1247. Un principe similaire était applicable en matière paulienne dans le droit antérieur - l'art. 1038 C.c.B.-C. étant considéré inapplicable aux paiements faits en fraude des droits d'un créancier; sur ce point, voir: supra, note 31 . Pour un aperçu du droit nouveau en matière de paiement préférentiel, voir: Baudouin, $4^{\mathrm{e}}$ éd., supra, note $3, \mathrm{n}^{\mathrm{0}} 683$. 
Par ailleurs, il est vrai que l'art. 1632 C.c.Q. évoque des situations où le tiers, conscient de certaines circonstances, peut effectivement s'avérer être de mauvaise foi. Ceci peut amener à croire qu'un article distinct, calqué sur l'art. 1038 C.c.Q., serait superflu. Or, il faut reconnaître que ce n'est pas toujours le cas.

Ainsi, il arrive que les faits ne permettent pas de recourir aux présomptions édictées à l'art. 1632 C.c.Q. - si le débiteur est toujours solvable ou encore si le tiers ignore l'insolvabilité (accomplie ou recherchée) de son cocontractant. Dans ce cas, seul le principe général de l'art. 1631 gouverne le recours du créancier. Dans cette hypothèse, le tiers souffre de l'absence d'un moyen de défense personnel. Il doit alors se rabattre sur la solution de rechange que constitue la preuve de bonne foi du débiteur - une preuve qui peut s'avérer impossible si, à son insu, le débiteur était effectivement animé par une intention frauduleuse.

En ce qui a trait aux situations où l'art. 1632 C.c.Q. trouve application, d'aucuns prétendront que les faits dont le tiers est informé l'empêchent de plaider sa bonne foi. La connaissance de l'insolvabilité, dans cette optique, est assimilée de façon irréfragable à de la mauvaise foi. Ce serait là oublier une distinction fondamentale: c'est la connaissance du préjudice causé aux autres créanciers qui équivaut à de la mauvaise foi, alors que la connaissance de l'insolvabilité $d u$ débiteur permet simplement de supposer que le tiers connaît également le caractère préjudiciable de l'acte. Si certaines situations ne permettent pas de tracer une telle ligne de démarcation ${ }^{47}$, il en est d'autres où la distinction est bien réelle $^{48}$. On peut notamment penser à l'institution financière qui, informée de l'insolvabilité de son cocontractant, accepte malgré tout de lui faire crédit dans le but de l'aider à corriger la situation. Ici, la situation du tiers de bonne foi est plus précaire encore. En effet, ce dernier ne dispose d'aucun moyen de défense personnel, à défaut d'indications en ce sens à l'art. 1632 et de l'absence d'un article distinct similaire à l'article 1038 C.c.B.-C. Qui plus est, le tiers est privé du moyen de défense additionnel que constituait, dans le droit antérieur, la preuve de bonne foi du débiteur, puisque l'article 1632 présume l'intention frauduleuse de façon absolue.

47. Ce serait le cas, par exemple, lorsque le tiers sait que le débiteur cherche à se rendre insolvable, ce qui équivaut plus ou moins à la connaissance d'un préjudice causé intentionnellement aux créanciers de son cocontractant.

48. Spenard c. Lapointe, supra, note 21, p. 16-18; Perreault c. Société de prêts et placements du Québec, (1935) C.S. 70; In re Callway Sash and Door Inc.: Perras c. Banque provinciale du Canada, supra, note 17. Voir aussi: Saulnier c. Evans, 192531 R.L.n.s. 383 (C.S.); Butler c. Castonguay, [1949] C.S. 52. 
La disparition de l'art. 1038 C.c.B.-C. contribue à rompre l'équilibre que le droit antérieur cherchait à maintenir entre le créancier victime des agissements de son débiteur et les tiers qui ont contracté, de bonne foi et à titre onéreux, avec ce dernier. Comme dans le cas de la présomption légale d'intention frauduleuse du débiteur - c'est-à-dire par une modification possiblement fortuite et certainement inopportune -, le droit nouveau offre une protection excessive des droits du créanciers au détriment de personnes qui ont pu conclure un acte en toute bonne foi.

L'action en inopposabilité peut affecter de nombreux tiers qui transigent régulièrement et de bonne foi avec un débiteur en difficulté. Les institutions financières sont de ce nombre, tout comme différents types de fournisseurs. Tous sont susceptibles, en acceptant de contracter à titre onéreux avec un débiteur traversant une période difficile, de contribuer à ses efforts en vue d'une meilleure posture financière. Certes, les cas de transactions frauduleuses existent. Pourtant, le droit ancien permettait, de façon relativement satisfaisante, d'exercer un contrôle dans de telles circonstances. Les tiers de bonne foi, déjà inconfortables en raison du caractère désormais irréfragable de la présomption d'intention frauduleuse, sont affectés de façon plus significative encore par la disparition de leur moyen de défense personnel. Il n'est donc pas exagéré d'anticiper, si ce n'est un mouvement de panique, du moins une très grande prudence dans leurs affaires courantes. La perspective d'une instabilité des rapports contractuels n'est certes pas de nature à favoriser un climat de confiance ${ }^{49}$.

Dans les premières analyses du droit nouveau, la question de la bonne foi du tiers semble être négligée au profit de celle portant sur le caractère irréfragable de la présomption établie à l'art. 1632 C.c.Q. Le fait de redonner à la présomption de l'art. 1632 un caractère réfragable - que ce soit par une modification législative ou une interprétation jurisprudentielle - ne suffirait pas à rétablir l'équilibre rompu par la réforme, puisque le tiers ne peut éviter l'inopposabilité lorsque le débiteur était effectivement de mauvaise foi. La solution la plus viable serait de faire renaître l'art. 1038 C.c.B.-C. Une telle modification rendrait au tiers de bonne foi un moyen de défense à toutes les actions en inopposabilité impliquant une transaction à titre onéreux, qu'elles soient ou non soumises à la présomption d'intention frauduleuse du débiteur. 


\section{Conclusion}

Un survol des dispositions nouvelles en matière d'action en inopposabilité permet de constater des modifications importantes en ce qui a trait à l'état d'esprit des parties à l'acte attaqué. Ces modifications qui, dans une certaine mesure, ne semblent pas même souhaitées par le législateur, pourraient avoir des répercussions importantes dans le cadre de contrats à titre onéreux et créer des situations fort injustes pour le débiteur ou le tiers contractant de bonne foi.

Il faut souhaiter que le législateur apporte les ajustements qui s'imposent. Quant à la nature de ces ajustements, il nous semble essentiel de bien distinguer les deux éléments qui apparaissent déficients dans le droit nouveau: énoncer clairement le caractère réfragable de la présomption d'intention frauduleuse du débiteur par l'emploi du mot «présumé» (conformément à l'art. 2847 C.c.Q.) et rétablir, dans un second alinéa de l'art. 1631 C.c.Q. ou dans un article distinct, la défense de bonne foi du tiers ${ }^{50}$.

Il existe certes des alternatives d'ordre judiciaire en cas de refus du législateur d'apporter de telles modifications. Il s'agit toutefois de simples palliatifs plus ou moins souhaitables selon le cas.

À l'égard de la présomption d'intention frauduleuse du débiteur, les tribunaux pourraient être tentés d'interpréter le mot «réputé» à la lumière du Code civil du Bas-Canada et d'ignorer par le fait même le sens donné par l'article 2847 C.c.Q. ${ }^{51}$ Une telle solution n'irait toutefois pas sans entraîner certaines interrogations, dans la mesure où elle porte atteinte à la volonté du législateur de rationaliser l'emploi de termes destinés à exprimer la nature de présomptions légales. Sans prêcher en faveur d'un positivisme étroit, il nous semble pour le moins inquiétant, dans un système de droit codifié, de passer outre à un texte aussi clair au motif que le législateur semble, dans le contexte d'un recours particulier, avoir ignoré la portée de cette règle.

Si le législateur juge à propos d'édicter une telle disposition, il ne nous semble pas souhaitable de le faire dans le corps de l'art. 1632, puisque ce dernier se limite aux cas où l'intention frauduleuse du débiteur découle de la présomption légale - ce qui priverait d'un moyen de défense le tiers qui contracte avec un débiteur solvable mais de mauvaise foi. Par ailleurs, la modification proposée soulèverait une nouvelle question, à savoir l'applicabilité de ce moyen de défense dans le cas d'un paiement et non d'un contrat; sur l'état du droit antérieur sur cette question, voir supra, note 31.

51. Dans cette optique, l'art. 2847 C.c.Q. ne constituerait qu'une règle d'interprétation, expression d'ailleurs utilisée dans la Loi d'application de la réforme du Code civil, L.Q. 1992, c. 57, art. 142: Bisson, supra, note 25, p. 18. Si les professeurs Bisson et Ducharme s'entendent sur le caractère inopportun de l'art. 2847 C.c.Q., le second va plus loin en proposant une modification de cet article et la disparition de la règle d'interprétation qui y est énoncée: Ducharme, supra, note 25, p. 45. 
La recherche d'une alternative permettant la reconnaissance d'un moyen de défense personnel au tiers de bonne foi s'avère plus ardue. Certains pourraient être tentés d'avancer qu'en l'absence d'une disposition similaire à l'art. 1038 C.c.B.-C., on en revient au principe selon lequel la bonne foi se présume ${ }^{51 a}$. Ce serait là oublier que le renversement de la présomption de bonne foi, en matière paulienne, ne découlait pas uniquement de cette disposition, mais aussi et surtout du fait que le comportement frauduleux du débiteur, démontré par preuve directe ou à l'aide de présomptions, permettait de considérer que les deux parties au contrat agissaient de mauvaise foi jusqu'à preuve du contraire. Par ailleurs, même en acceptant un tel argument aux fins de discussion, il ne viendrait rien modifier à la position du tiers de bonne foi puisque, selon nous, la mauvaise foi du tiers ne constitue pas une condition de l'action en inopposabilité mais plutôt un moyen de défense ${ }^{52}$.

L'effort de clarification et de systématisation du législateur est mis en péril par ce qui apparaît comme des modifications involontaires ou dont les répercussions ont été sous-estimées. C'est pourquoi il nous semble souhaitable qu'il fasse marche arrière pour revenir à une position dont il n'a, en définitive, jamais cherché à s'éloigner. à l'égard du tiers n'intervient que lorsque la présomption de l'art. 1035 trouve application; pour une illustration récente de cette tendance, voir: Meunerie Camirand Inc. c. Banque Nationale du Canada, supra, note 35, p. 3-4. Cette position se défend fort bien au niveau pratique, puisque la connaissance de l'insolvabilité du débiteur place le tiers dans une situation plus compromettante que s'il ignore cette insolvabilité ou encore s'il sait ce débiteur solvable. Néanmoins, il nous semble plus difficile de justifier cette position au plan théorique étant donné que le Code ne distingue pas, l'art. 1038 C.c.B.-C. s'appliquant peu importe le mode par lequel l'intention frauduleuse du débiteur est prouvée.

52. Voir les autorités citées supra, note 36. Il serait théoriquement possible de contourner cette objection en considérant que l'art. 1631 C.c.Q. exige que les deux parties au contrat attaqué aient eu une intention frauduleuse. Cette position poserait néanmoins un problème majeur, soit celui de permettre au tiers de bonne foi d'éviter l'inopposabilité de l'acte même dans le cadre d'un contrat à titre gratuit. 